OPEN ACCESS

Edited by:

Aida Salameh,

Leipzig University, Germany

Reviewed by:

Hansen Chen,

Stanford University, United States

Rasim Mogulkoc,

Selçuk University, Turkey

Ming He,

Nanchang University, China

${ }^{*}$ Correspondence:

Ya L

yr4249@126.com

Specialty section:

This article was submitted to

Cardiovascular and

Smooth Muscle Pharmacology,

a section of the journal

Frontiers in Pharmacology

Received: 10 August 2020 Accepted: 06 November 2020

Published: 11 January 2021

Citation:

Niu J, Li Y, Song X, LiU Y, Li Y and Li Y (2021) Cardioprotective Effect of Echinatin Against Ischemial

Reperfusion Injury: Involvement of Hippo/Yes-Associated

Protein Signaling.

Front. Pharmacol. 11:593225. doi: $10.3389 /$ fphar.2020.593225

\section{Cardioprotective Effect of Echinatin Against Ischemia/Reperfusion Injury: Involvement of Hippo/Yes-Associated Protein Signaling}

\author{
Jieting $\mathrm{Niu}^{1}$, Yanguang $\mathrm{Li}^{2}$, Xiang Song ${ }^{2}$, Yunfeng $L i u^{1}$, Ying $L i^{1}$ and $Y a L i^{3 *}$ \\ ${ }^{1}$ Department of Geriatrics, Cangzhou Central Hospital, Cangzhou, China, ${ }^{2}$ Department of Thoracic Surgery, Cangzhou Central \\ Hospital, Cangzhou, China, ${ }^{3}$ Department of Cardiology, Cangzhou Central Hospital, Cangzhou, China
}

Background: Echinatin (Ech) has been reported to exert antioxidant and antiinflammatory activities. In this study, we aimed to characterize the functional role of Ech in myocardial ischemic/reperfusion (MI/R) injury and elucidate its underlying mechanism of action.

Method: We established in vivo and in vitro models of $\mathrm{Ml} / \mathrm{R}$ injury to determine the effect of Ech on Ml/R injury. Gene expression was examined using quantitative real-time polymerase chain reaction and western blotting. Myocardial infarction was assessed using tetrazolium chloride staining and the degree of myocardial injury was evaluated by measuring lactate dehydrogenase $(\mathrm{LDH})$ and creatine kinase-myocardial band (CK-MB) levels. Cell apoptosis was detected using the terminal deoxynucleotidyl transfer-mediated dUTP nick end-labeling (TUNEL) assay. The viability of H9c2 cells was determined using Cell Counting Kit-8 assay.

Results: MI/R induced myocardial infarction, which was mitigated by Ech treatment. Moreover, Ech treatment resulted in a marked decline of LDH and CK-MB levels in the serum and myocardium of $\mathrm{Ml} / \mathrm{R}$ rats. Ech treatment also restrained cardiomyocyte apoptosis in vivo and in vitro, as evidenced by reduction in LDH release, the number of TUNEL-positive cells, and caspase-3 activity. Furthermore, Ech administration inhibited $\mathrm{MI} / \mathrm{R}$-induced activation of Hippo/Yes-associated protein signaling in vivo and in vitro, as indicated by inhibition of mammalian sterile 20-like protein kinase 1, large tumor suppressor one, and YAP phosphorylation and promotion of YAP nuclear translocation. However, silencing of YAP counteracted the protective effect of Ech on hypoxia/reoxygenation-induced myocardial injury in vitro.

Conclusion: Ech exerted its protective effect against MI/R injury at least partially by suppressing the Hippo/YAP signaling pathway, providing novel insights into the remission of $\mathrm{Ml} / \mathrm{R}$ injury.

Keywords: echinatin, myocardial ischemia/reperfusion injury, hippo/yes kinase-associated protein signaling, caspase-3, lactate dehydrogenase 


\section{INTRODUCTION}

Myocardial infarction, resulting from the interruption of blood supply, is one of the most frequent cardiovascular emergencies, which remains to be the major cause of mortality and disability worldwide (Thygesen et al., 2018). To date, restoration of blood flow in the infarcted coronary artery as soon as possible is considered as the most effective treatment for myocardial infarction (Anderson and Morrow, 2017). However, restoration of coronary circulation may also aggravate reversible myocardial ischemia injury and lead to irreversible myocardial injury, termed as myocardial ischemic/reperfusion (MI/R) injury (Ferrari et al., 2017). MI/R injury is associated with poor clinical outcomes in patients with myocardial infarction and causes multiple cardiac complications. $\mathrm{MI} / \mathrm{R}$ is generally recognized as the serious pathophysiologic mechanism of myocardial infarction (Reed et al., 2017). In view of this, reducing $\mathrm{MI} / \mathrm{R}$ injury is necessary to reduce the myocardial infarct size and improve myocardial function.

Among these, glycyrrhiza has attracted much attention from researchers in the last few years. It has been shown to exhibit multifarious pharmacological activities, such as anti-myocardial infarction, anti-tumor, and anti-viral capabilities (Ojha et al., 2013). Glycyrrhiza contains many kinds of bioactive constituents, such as isoliquiritigenin, glycyrrhetinic acid, and licochalcone D., which have protective effects against MI/R injury (Zhang et al., 2013; Wu et al., 2015; Yuan et al., 2015). Echinatin (Ech), a characteristic retrochalone present in glycyrrhiza, has been found to possess several pharmacological activities (Ji et al., 2016). For example, Ech promoted the apoptosis of esophageal squamous cell carcinoma cells via induction of reactive oxygen species (ROS) production or endoplasmic reticulum stress and activation of p38 mitogen-activated protein kinase/c-Jun $\mathrm{N}$-terminal kinase signaling (Kwak et al., 2019). Furthermore, Ech treatment repressed epidermal growth factor receptor and mesenchymal-epithelial transition, induced ROS generation, and depolarized the mitochondria membrane potential, thereby inducing the apoptosis of non-small-cell lung cancer cells sensitive or resistant to gefitinib (Oh et al., 2020). Remarkably, the functional role of Ech in MI/R injury has not been well explored so far.

In this study, we aimed to characterize the functional role of Ech in the progression of MI/R injury, as well as elucidate the potential underlying molecular mechanisms. Our results demonstrated that Ech mitigated MI/R injury at least partially by suppressing Hippo/Yes kinase-associated protein (YAP) signaling, thus implying the protective effect of Ech on MI/R injury and providing an experimental basis for its clinical use.

\section{MATERIALS AND METHODS}

\section{In Vivo Model of Myocardial Ischemic/ Reperfusion Injury}

Male Wister rats (8 weeks old, weighing 250-280 g) from HuaFuKang (Beijing, China) were bred in laboratory settings and fed standard powdered rat diet and tap water. This study was approved by the Animal Care and use Committee of the Cangzhou Central Hospital. Rats were randomly divided into three groups: 1) Sham group, 2) MI/R group, and 3) MI/R + Ech group. After fasting overnight, all rats were operated following intraperitoneal injection of anesthesia with pentobarbitone $(50 \mathrm{mg} / \mathrm{kg})$. In the $\mathrm{MI} / \mathrm{R}$ group, the heart was exposed by thoracic surgery. A 6-0 silk ligature was passed beneath the left anterior descending coronary artery (LAD). The successfully established MI rat model showed significant elevation of ST segment and presented a color change of myocardial tissue. After $30 \mathrm{~min}$ of occlusion, the LAD was reperfused for $24 \mathrm{~h}$ by opening the ligation. The same procedures were followed for the sham-operated rats, except the suture was left untied. The rats in the MI/R + Ech group were intraperitoneally injected with either vehicle or Ech $(20,40$, and $80 \mathrm{mg} / \mathrm{kg}$; purity $\geq 98 \%$; Chengdu Must Biotechnology, Chengdu, China) $10 \mathrm{~min}$ before reperfusion. Ech was dissolved in DMSO and then diluted with phosphate buffer saline. The final concentration of DMSO was 1\%, and the injection volume of each rat was $4 \mathrm{ml}$. During surgical procedures, the body temperature of rats was continuously monitored by a rectal thermometer. We kept the body temperature of rats at $37.0 \pm 0.5^{\circ} \mathrm{C}$ using a heating pad.

\section{Tetrazolium Chloride Staining}

The animals were sacrificed after $24 \mathrm{~h}$ of reperfusion. Following this, the hearts were quickly resected, frozen in liquid nitrogen, and then cut into $2 \mathrm{~mm}$ thick sections. These sections were incubated with $1 \%$ TTC solution (Solarbio, Beijing, China) for 30 min away from the light. The infarcted tissues were white, while the viable tissues were red. Subsequently, these sections were fixed in $10 \%$ formalin, followed by analysis using Image software (NIH, Rockville, MD, United States). The infarct size was calculated as the proportion of infarct area (white) to the total non-infract area (red).

\section{Evaluation of Lactate Dehydrogenase and Creatine Kinase-Myocardial Band Levels}

At $24 \mathrm{~h}$ post reperfusion, blood sample collection was performed before the rats were sacrificed. The serum was collected after 30 min of blood clotting at $25^{\circ} \mathrm{C}$. The myocardium was also removed rapidly after the rats were sacrificed. The myocardium was washed, weighed, and then homogenized in phosphate buffered saline. Following centrifugation, the supernatants were carefully collected for all subsequent experiments. Next, the serum and supernatants were assayed for $\mathrm{LDH}$ and $\mathrm{CK}-\mathrm{MB}$ levels using commercially-available enzyme-linked immunosorbent assay kits (Nanjing Jiancheng Bioengineering Institute, Nanjing, China) following the manufacturer's instructions.

Myocardial damage was also evaluated by measuring $\mathrm{LDH}$ release using the LDH release Assay Kit (Beyotime, Shanghai, China). Briefly, H9c2 cells were harvested after Ech treatment, and then cultured with $\mathrm{LDH}$ release reagent for $1 \mathrm{~h}$. Next, the supernatant was collected after centrifugation of $\mathrm{H} 9 \mathrm{c} 2$ cells, followed by incubation with $\mathrm{LDH}$ detection reagent for $30 \mathrm{~min}$ 
in the dark. The release of LDH was analyzed by determination of the absorbance of each sample at $490 \mathrm{~nm}$.

\section{Terminal Deoxynucleotidyl Transfer-Mediated dUTP Nick End-Labeling Assay}

A One Step TUNEL Apoptosis Assay Kit (Beyotime) was utilized to evaluate cardiomyocyte apoptosis. In brief, tissue sections and cell smears were fixed for $30 \mathrm{~min}$ with $4 \%$ paraformaldehyde solution. After permeation with $0.3 \%$ Triton X-100, the samples were reacted with TUNEL detection solution for $1 \mathrm{~h}$ away from the light, following by staining with $4^{\prime}, 6$-diamidino-2phenylindole. Following sealing with antifade mounting medium, the samples were analyzed under a fluorescence microscope (Olympus, Tokyo, Japan). The apoptosis of cardiomyocyte was assessed by determining the ratio of the number of TUNEL-positive cells to the number of total cells.

\section{Determination of Caspase-3 Activity}

Cell apoptosis was assessed by determination of caspase- 3 activity using a caspase- 3 activity assay kit (Beyotime), following the product manual. The myocardium and H9c2 cells were collected, lyzed, and centrifuged for $15 \mathrm{~min}$, and the supernatants were collected. A Bradford Protein Assay kit (Bio-Rad, Hercules, CA, United States) was utilized to examine the concentration of extracted protein. The supernatants were reacted with AcDEVD-pNA for $1 \mathrm{~h}$ at $37^{\circ} \mathrm{C}$ and the absorbance was measured at $405 \mathrm{~nm}$.

\section{Quantitative Real-Time Polymerase Chain Reaction}

RNA isolation was carried out using the TRIzol kit (Beyotime) as recommended by the manufacturer. cDNA synthesis was performed using PrimeScript ${ }^{\mathrm{TM}}$ RT Enzyme Mix I (Takara, Dalian, China). qRT-PCR analysis was performed using SYBR $^{\circledR}$-Green PCR Master Mix (Takara). The expression of YAP was analyzed by the $2^{-\Delta \Delta C T}$ method using glyceraldehyde-3-phosphate dehydrogenase (GAPDH) as a housekeeping gene.

\section{Western Blotting}

Total protein was isolated with radioimmunoprecipitation buffer and then quantified using the BCA protein assay kit (Solarbio), as per the manufacturer's instructions. Cytoplasmic and nuclear proteins were isolated using the Nuclear and Cytoplasmic Protein Extraction Kit (Boster, Beijing, China) according to the manufacturer's recommendations. After sodium lauryl sulfatepolyacrylamide gel electrophoresis, the isolated proteins were transferred onto polyvinylidene difluoride membranes. The membranes were blocked with $5 \%$ non-fat milk and then blotted with the following antibodies from Abcam (Cambridge, MA, United States) or Cell Signaling Technology (Beverly, MA, United States): anti-phosphorylated (p)mammalian sterile 20-like protein kinase 1 (MST1) antibody
(1:1,000), anti-p-large tumor suppressor 1 (LATS1) antibody (1: $1,000)$, anti-p-YAP antibody (1:2,000), anti-YAP antibody (1: $1,000)$, anti-H3 antibody (1:1,000), and anti-GAPDH antibody $(1: 3,000)$ at $4{ }^{\circ} \mathrm{C}$ overnight. GAPDH and histone $\mathrm{H} 3$ were used internal loading controls. Following $1 \mathrm{~h}$ of incubation with horseradish peroxidase-labeled secondary antibody (1:2,000; Abcam), blots were developed with an enhanced chemiluminescence kit (Beyotime) as per the manufacturer's recommendations.

\section{In Vitro Model of Myocardial Ischemic/ Reperfusion Injury}

H9c2 cells were purchased from the Cell Bank of the Chinese Academy of Sciences (Shanghai, China) and then cultured in Dulbecco's modified Eagle's medium (DMEM) supplemented with $5 \%$ fetal bovine serum and $1 \%$ penicillin-streptomycin in a humidified $5 \% \mathrm{CO}_{2}$ incubator at $37^{\circ} \mathrm{C}$. To establish an in vitro model of $\mathrm{MI} / \mathrm{R}$ injury, $\mathrm{H} 9 \mathrm{c} 2$ cells were cultured for $4 \mathrm{~h}$ in glucose- and serum-free medium under $95 \% \mathrm{~N}_{2} / 5 \% \mathrm{CO}_{2}$ at $37^{\circ} \mathrm{C}$. When the oxygen content is more than $1 \%, \mathrm{~N}_{2}$ will be automatically charged into the incubator to always maintain the oxygen concentration of the incubator at the set value. Next, cells were cultured for $24 \mathrm{~h}$ in DMEM plus Ech (10 or $20 \mu \mathrm{m})$ at $37^{\circ} \mathrm{C}$ in a humidified incubator with $5 \% \mathrm{CO}_{2}$ and $95 \%$ air. $\mathrm{H} 9 \mathrm{c} 2$ cells were then harvested for subsequent experiments.

Small interfering RNA specific for YAP (si-YAP) and sinegative control (NC) were purchased from GenePharma (Shanghai, China). Cell transfection was performed using Lipofectamine $^{\mathrm{TM}} 2000$ (Invitrogen, Carlsbad, CA, United States) as per the instruction manual. The knockdown efficiency was affirmed using qRT-PCR and western blot analyses.

\section{Cell Counting Kit-8 Assay}

H9c2 cells were seeded into a 96-well plate and cultured overnight in a humidified incubator at $37^{\circ} \mathrm{C}$ with $5 \% \mathrm{CO}_{2}$. Following this, cells were exposed to different doses $(0,1,5,10,20,40$, and $80 \mu \mathrm{m})$ of Ech for $24 \mathrm{~h}$. Following the addition of CCK- 8 solution (Solarbio), $\mathrm{H} 9 \mathrm{c} 2$ cells were grown in a $5 \% \mathrm{CO}_{2}$ incubator at $37^{\circ} \mathrm{C}$ for $2 \mathrm{~h}$. The absorbance of each sample at $450 \mathrm{~nm}$ was analyzed using a microplate reader.

\section{Statistical Analysis}

All results are presented as mean \pm standard deviation of three independent experiments. Statistical analysis was performed using one-way analysis of variance with post-hoc Bonferroni correction using SPSS 20.0 software (SPSS Inc., Chicago, IL, United States). Statistical significance was set at $p<0.05$.

\section{RESULTS}

\section{Ech Mitigates I/R-Induced Myocardial Injury In Vivo}

To examine the protective effect of Ech on MI/R injury, MI/R rats were subjected to Ech $(20,40$, and $80 \mathrm{mg} / \mathrm{kg})$ treatment before 

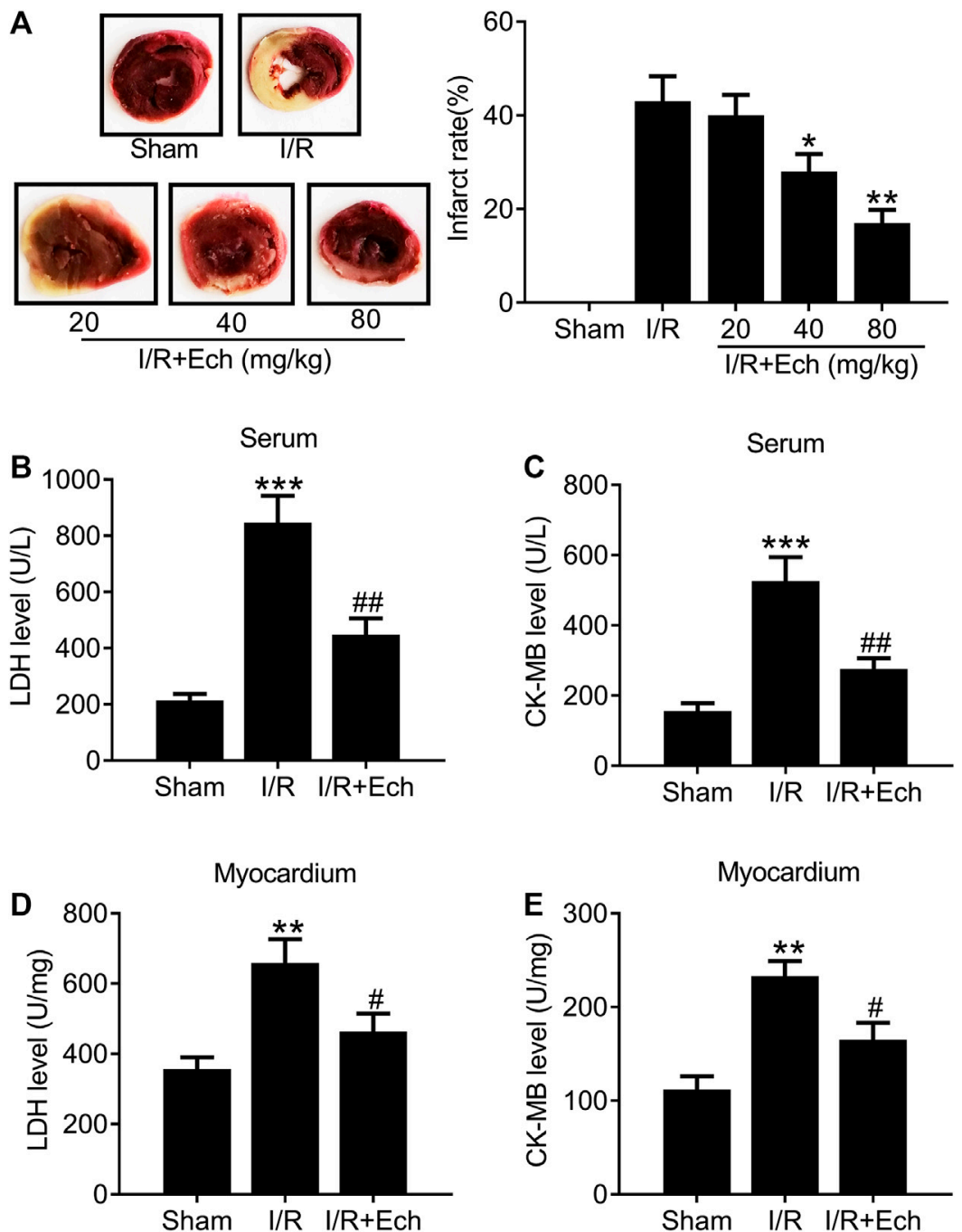

FIGURE 1 | Ech mitigates I/R-induced myocardial injury in vivo. MI/R rats were subjected to Ech (20, 40, and 80 mg/ $/ \mathrm{kg}$ ) treatment before reperfusion. (A) Representative heart sections stained with $\Pi \mathrm{TC}$ after MI/R and Ech administration. Myocardial infarction was measured as the proportion of infarct area (white) to the total non-infract area $(\mathrm{red})(n=5)$. (B, C) The degree of myocardial injury was assessed by measurement of serum LDH and CK-MB levels ( $n=5)$. (D, E) Measurements of myocardial LDH and CK-MB levels in the heart of rats $(n=5) .{ }^{*} p<0.05$ and ${ }^{* \star} p<0.01$ compared with $\mathrm{MI} / \mathrm{R}$ rats.

reperfusion. After $24 \mathrm{~h}$ of reperfusion, the myocardial infarct size was measured. As shown in Figure 1A, MI/R induced myocardial infarction relative to the sham group. However, Ech administration markedly reduced MI/R-induced myocardial infarction. Considering the significant cardioprotective effect of Ech at $80 \mathrm{mg} / \mathrm{kg}$, we chose this concentration for all following experiments. MI/R rats also showed a significant increase in the levels of serum $\mathrm{LDH}$ and $\mathrm{CK}-\mathrm{MB}$ relative to the sham group; however, Ech treatment attenuated MI/R-induced elevation of $\mathrm{LDH}$ and CK-MB serum levels (Figures 1B,C). Similarly, MI/R increased the levels of myocardial $\mathrm{LDH}$ and $\mathrm{CK}-\mathrm{MB}$ in the heart of rats, which was inhibited by Ech treatment (Figures 1D,E).

\section{Ech Inhibits Cardiomyocyte Apoptosis in Myocardial Ischemic/Reperfusion Rats}

Next, we evaluated the cardioprotective effect of Ech against MI/ R-induced cardiomyocyte apoptosis. Compared to shamoperated rats, $\mathrm{MI} / \mathrm{R}$ rats showed an increase in the number of TUNEL-positive cells, as determined by TUNEL assay. However, Ech treatment significantly inhibited MI/R-induced cardiomyocyte apoptosis (Figures 2A,B). To clarify the mechanism by which Ech protects against MI/R-induced cardiomyocyte apoptosis, the effect of Ech treatment on caspase-3 activity was further examined in the cardiac tissues of $\mathrm{MI} / \mathrm{R}$ rats. Results showed that MI/R significantly enhanced caspase-3 activity; however, this effect was blocked by Ech treatment (Figure 2C). 

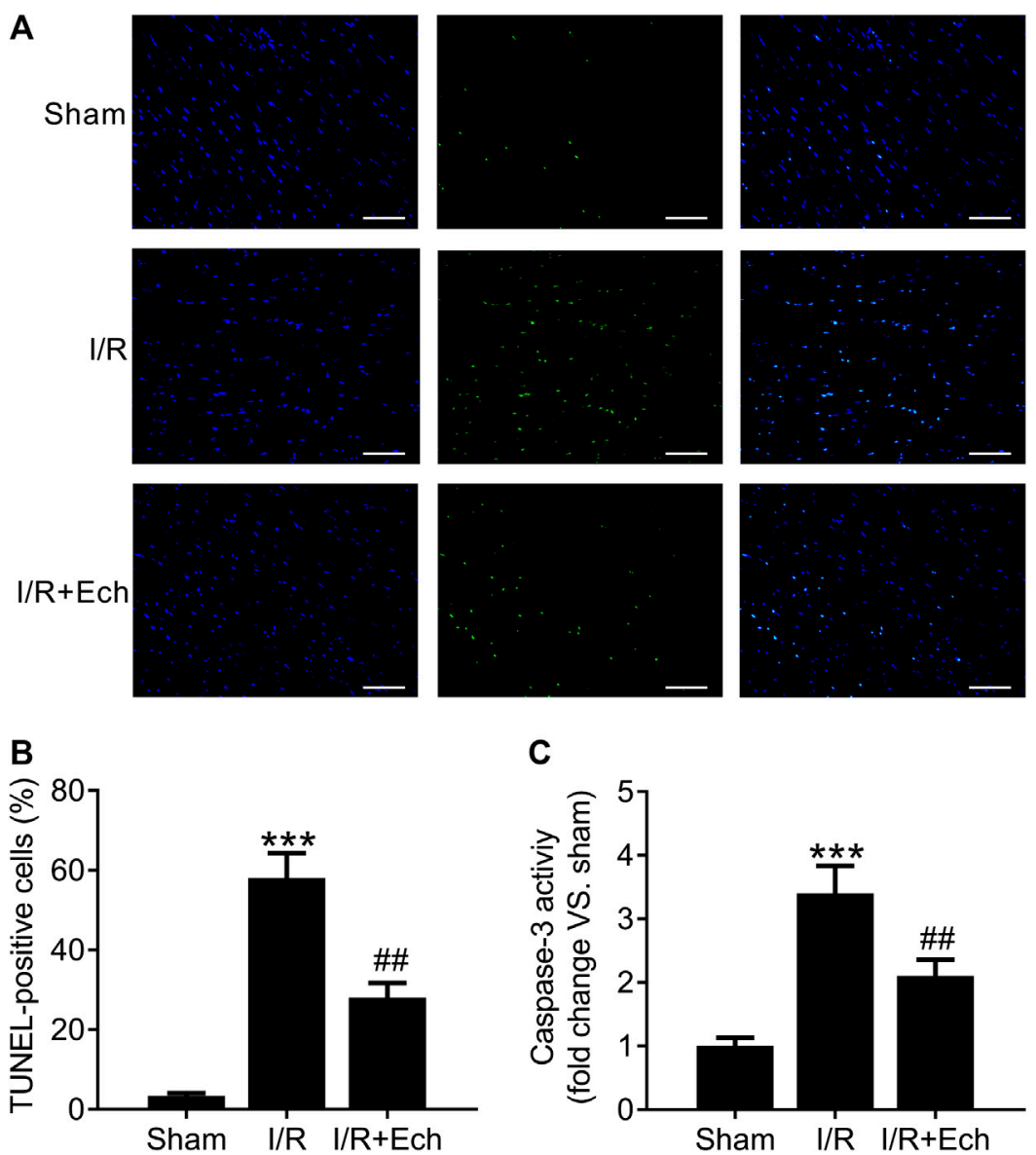

FIGURE 2 | Ech inhibits cardiomyocyte apoptosis in MI/R rats. (A) Representative images and (B) quantification of TUNEL staining indicated that Ech administration mitigated MI/R-induced cardiomyocyte apoptosis $(n=4)$. (C) Analysis of caspase-3 activity showed that the increase in caspase-3 activity in MI/R rats was reversed by Ech treatment $(n=4) .{ }^{* *} p<0.001$ compared with the sham group; ${ }^{\# \#} p<0.01$ compared with $\mathrm{MI} / \mathrm{R}$ rats.

\section{Ech Inhibits the Hippo/YAP Signaling Pathway In Vivo}

To explore whether the Hippo/YAP signaling pathway mediates the protective effect of Ech on MI/R injury, we detected the protein expression levels of p-MST1, p-LATS1, $\mathrm{p}-\mathrm{YAP}$, and YAP using western blotting. Results showed that the protein levels of p-MST1, p-LATS1, and p-YAP were elevated, but those of YAP were reduced in the cardiac tissues of $\mathrm{MI} / \mathrm{R}$ rats compared to sham-operated rats. However, administration of Ech significantly reduced the levels of p-MST1, p-LATS1, and p-YAP and increased the levels of YAP relative to the MI/R group (Figure 3A). Besides, our results demonstrated that MI/R restrained YAP nuclear translocation, which was reversed by Ech treatment. This was also affirmed by obviously lower nuclear to cytoplasmic ratio of YAP protein level in MI/R rats compared to sham-operated rats, and markedly higher nuclear to cytoplasmic ratio of YAP protein level in Ech-treated $\mathrm{MI} / \mathrm{R}$ rats relative to $\mathrm{MI} / \mathrm{R}$ rats (Figure 3B).

\section{Ech Protects H9c2 Cells Against Hypoxia/ Reoxygenation-Induced Myocardial Damage}

To further confirm the cardioprotective effect of Ech on MI/R injury, we established an in vitro model of MI/R injury by exposing H9c2 cells to H/R. As determined by CCK-8 assay, 40 and $80 \mu \mathrm{m}$ of Ech markedly restrained the viability of H9c2 cells compared to the controls, but $1,5,10$, and $20 \mu \mathrm{m}$ of Ech did not influence H9c2 cell viability (Figure 4A). Therefore, 10 and $20 \mu \mathrm{m}$ of Ech were selected for subsequent experiments. H9c2 cells subjected to $\mathrm{H} / \mathrm{R}$ condition showed a marked decline in cell viability; however, this effect was blocked by treatment with 10 and $20 \mu \mathrm{m}$ Ech (Figure 4B). Consistently, a marked elevation in $\mathrm{LDH}$ release was observed in $\mathrm{H} 9 \mathrm{c} 2$ cells exposed to $\mathrm{H} / \mathrm{R}$, and this effect was abolished following Ech $(10$ and $20 \mu \mathrm{m})$ treatment (Figure 4C). In parallel, H/R promoted H9c2 cell apoptosis, as indicated by the increased number of TUNEL-positive cells. However, Ech at concentrations of 10 and $20 \mu \mathrm{m}$ attenuated H/R-induced cell apoptosis (Figure 4D). Moreover, H/R 


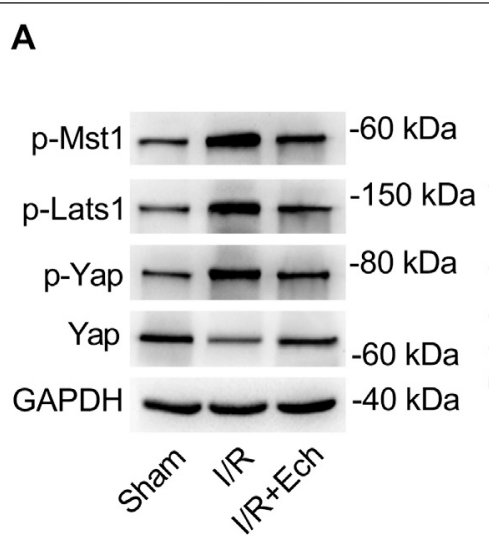

B

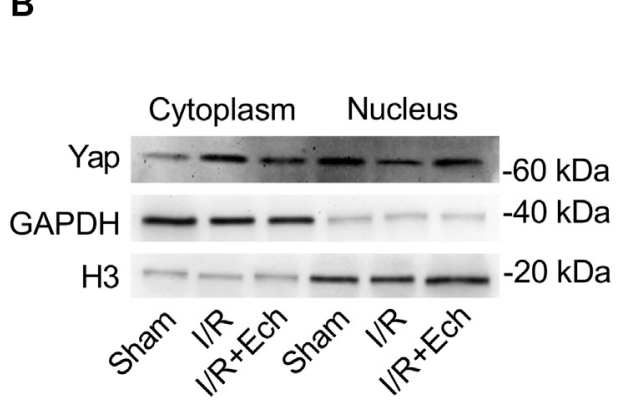

\section{- Sham $\approx \mathrm{l} / \mathrm{R}$ 曰 I/R+Ech}
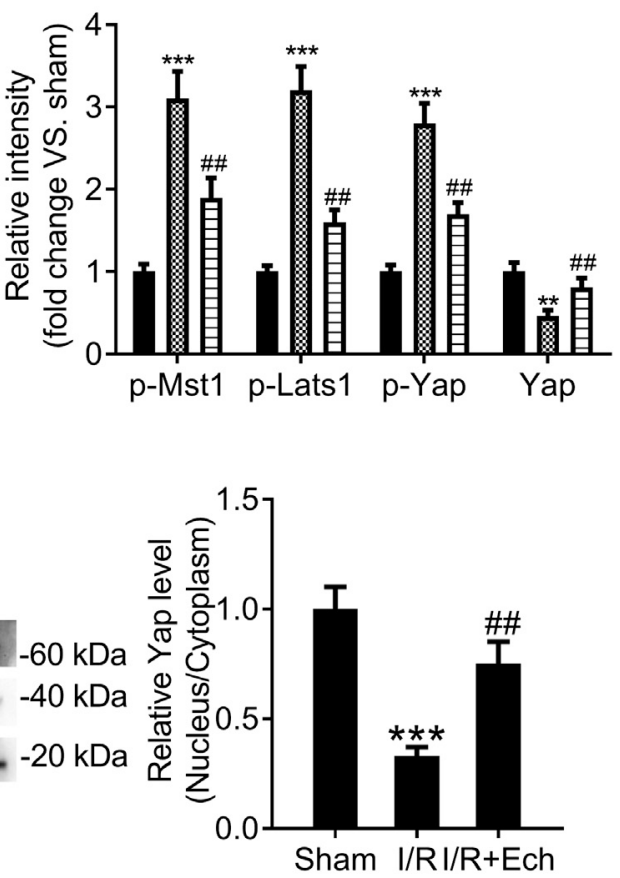

FIGURE 3 | Ech inhibits the Hippo/YAP signaling pathway in vivo. (A) Representative images of western blot analysis and quantification of p-MST1, p-LATS1, p-YAP, and YAP expression in the cardiac tissues of MI/R rats $(n=4)$. (B) Representative images of western blot analysis and quantification of cytoplasmic and nuclear YAP expression in the cardiac tissues of Ml/R rats $(n=4) .{ }^{* \star} p<0.01$ and ${ }^{\star \star *} p<0.001$ compared with the sham group; ${ }^{\# \#} p<0.01$ compared with MI/R rats.

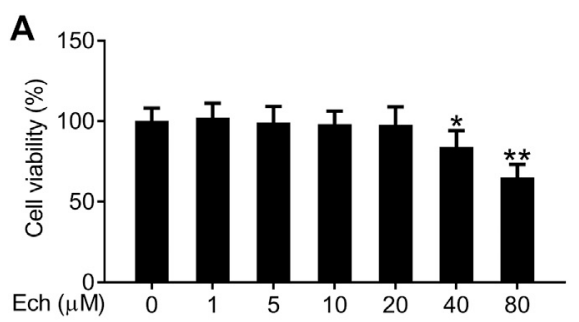

C

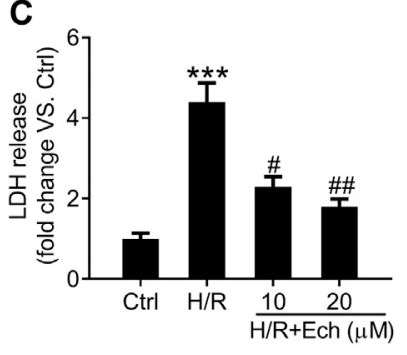

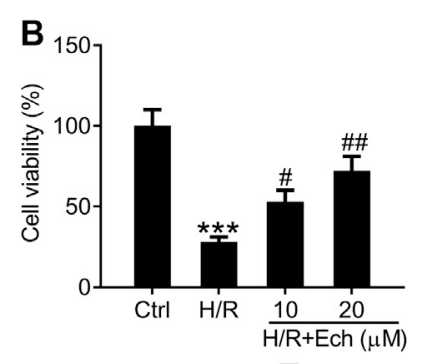

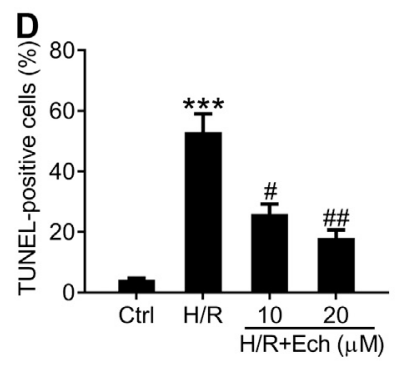

E

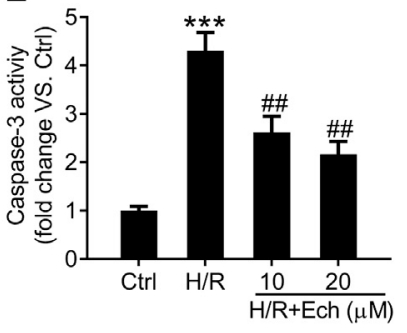

FIGURE 4 | Ech protects H9c2 cells against H/R-induced injury. H9c2 cells were exposed to increasing doses (0, 1, 5, 10, 20, 40, and 80 $\mu$ m) of Ech. (A) The cytotoxicity of Ech in $\mathrm{H} 9 \mathrm{c} 2$ cells was evaluated using CCK-8 assay. H9c2 cells were exposed to H/R and then treated with Ech (10 and 20 mm), ( $n=3$ ). (B) CCK-8 assay results showing the protective effect of Ech on $\mathrm{H} / \mathrm{R}$-induced inhibition of $\mathrm{H} 9 \mathrm{c} 2$ cell viability $(n=3)$. (C) Results showing the inhibitory effect of Ech on $\mathrm{H} / \mathrm{R}$-induced LDH release $(n=3)$. (D) Representative images and quantification of TUNEL staining showing the protective effect of Ech on H/R-induced H9c2 cell apoptosis ( $n=3)$. (E) Analysis of caspase- 3 activity showing the reduced activity of caspase- 3 in $\mathrm{H} 9 \mathrm{c} 2$ cells treated with $\mathrm{H} / \mathrm{R}$ and Ech relative to cells treated with $\mathrm{H} / \mathrm{R}$ only ( $n=3$ ). ${ }^{*} p<0.05$, ${ }^{* *} p<0.01$, and ${ }^{\star * *} p<0.001$ compared with the control (Ctrl) group; ${ }^{\#} p<0.05$ and ${ }^{\# \#} p<0.01$ compared with the H/R group. 

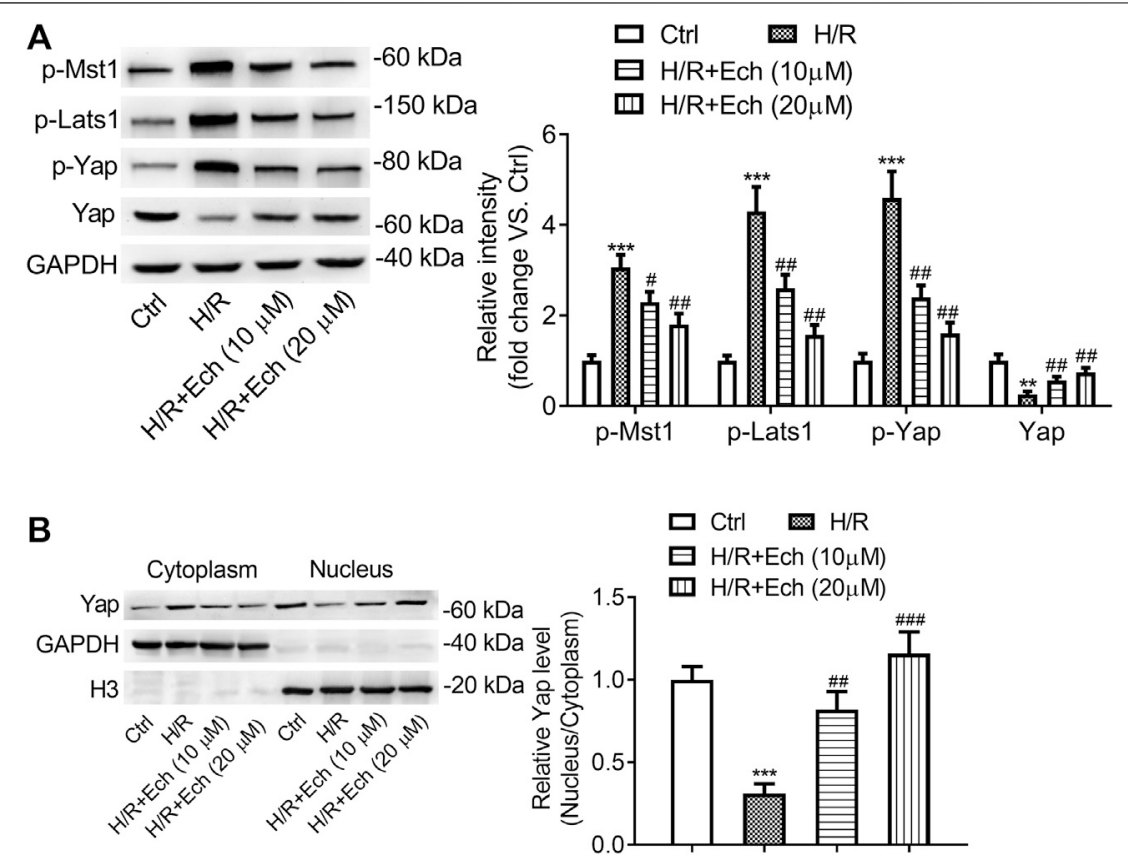

FIGURE 5 | Ech restrains phosphorylation of MST1 and LATS1, and triggers YAP nuclear translocation in vitro. H9c2 cells were subjected to H/R and then treated with Ech $(10$ and $20 \mu \mathrm{m})$. (A) Representative images of western blot analysis and quantification of p-MST1, p-LATS1, p-YAP, and YAP expression in H9c2 cells $(n=3)$. (B) Representative images of western blot analysis and quantification of cytoplasmic and nuclear YAP expression in H9c2 cells $(n=3) .{ }^{* *} p<0.01$ and ${ }^{* \star *} p<0.001$ compared with the Ctrl group; ${ }^{\#} p<0.05,{ }^{\# \#} p<0.01$, and ${ }^{\# \#} p<0.001$ compared with the $H / R$ group.

exposure increased caspase-3 activity in H9c2 cells, and this effect was counteracted by Ech administration (Figure 4E).

\section{Ech Restrains Phosphorylation of MST1 and LATS1, and Triggers YAP Nuclear Translocation In Vitro}

To determine the impact of Ech on Hippo/YAP signaling in vitro, H9c2 cells were exposed to H/R, followed by treatment with Ech $(10$ and $20 \mu \mathrm{m})$. As determined by western blot analysis, the expression level of p-MST1, p-LATS1, and p-YAP was elevated, but that of YAP was reduced in H/R-treated H9c2 cells; however, these effects were abolished by administration of Ech (Figure 5A). In addition, our results showed that exposure to $\mathrm{H} / \mathrm{R}$ inhibited YAP nuclear translocation, as evidenced by a decrease in the nuclear to cytoplasmic ratio of YAP protein. However, this effect was blocked by Ech administration (Figure 5B).

\section{Loss of YAP Counteracts the Protective Effect of Ech on Hypoxia/ Reoxygenation-Induced Myocardial Cell Injury In Vitro}

In order to determine whether silencing of YAP counteracts the protective effect of Ech on H/R-induced myocardial cell injury in vitro, si-YAP and si-NC were transfected into H9c2 cells and the silencing efficiency was evaluated by qRT-PCR assay. As expected, YAP expression was much lower in the si-YAP group than in the si-NC group (Figure 6A). Western blot analysis showed similar results (Figure 6B). Moreover, we found that silencing of YAP could abrogate the protective effect of Ech on $\mathrm{H} / \mathrm{R}$-induced inhibition of $\mathrm{H} 9 \mathrm{c} 2$ cell viability (Figure 6C). Knockdown of YAP also abolished Ech-mediated inhibition of $\mathrm{LDH}$ release in $\mathrm{H} 9 \mathrm{c} 2$ cells exposed to $\mathrm{H} / \mathrm{R}$ (Figure 6D). Furthermore, the results of TUNEL assay revealed that the protective effect of Ech on H/R-induced H9c2 cell apoptosis was hindered following si-YAP transfection, as indicated by the increase in number of TUNEL-positive cells (Figure 6E). Likewise, Ech treatment decreased caspase-3 activity in H/R-induced H9c2 cells, and this effect was counteracted by YAP silencing (Figure 6F).

\section{DISCUSSION}

The mechanism of MI/R injury is complex and involves multiple biological processes, including cardiomyocyte loss, which plays a vital role (Wu et al., 2018). Myocardial ischemia initiates multiple deleterious cascades, such as oxidative stress, autophagy, and inflammatory response, and then aggravates cardiomyocyte injury, contributing to contractile dysfunction and apoptosis of cardiomyocytes (Granger and Kvietys, 2015; Kurian et al., 2016). Damage of mitochondrial membrane integrity causes release of cytochrome $c$, which in turn triggers the activation of caspase- 3 and finally results in cardiomyocyte apoptosis (Badalzadeh et al., 
A

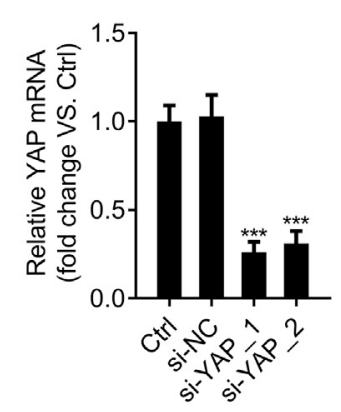

C
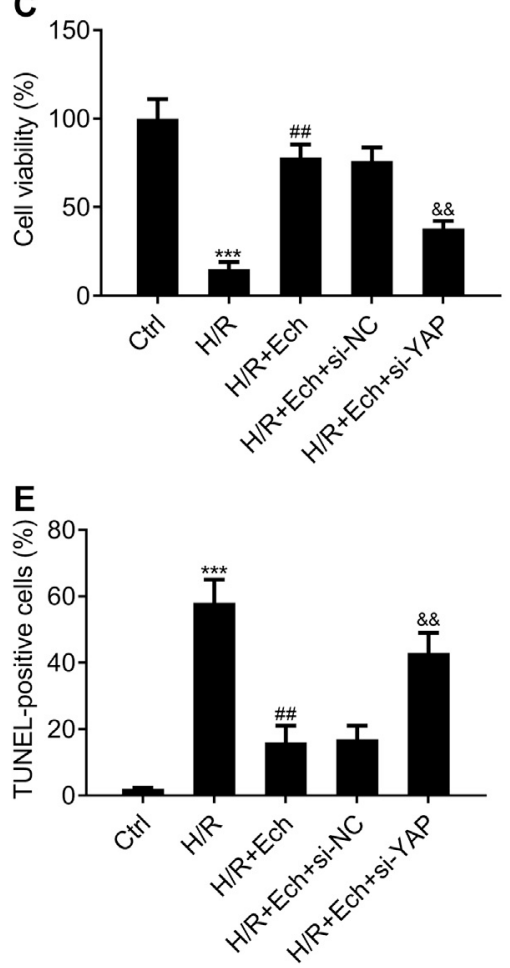

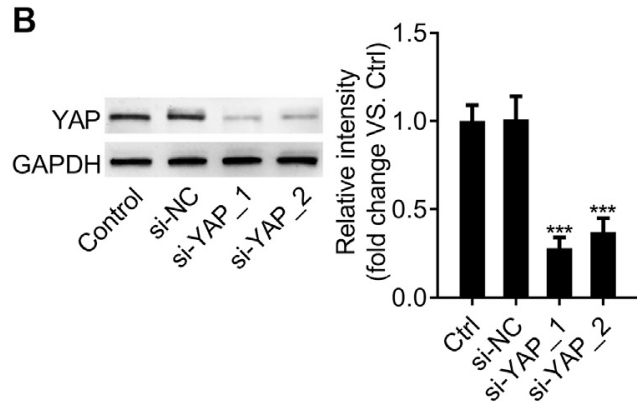

D

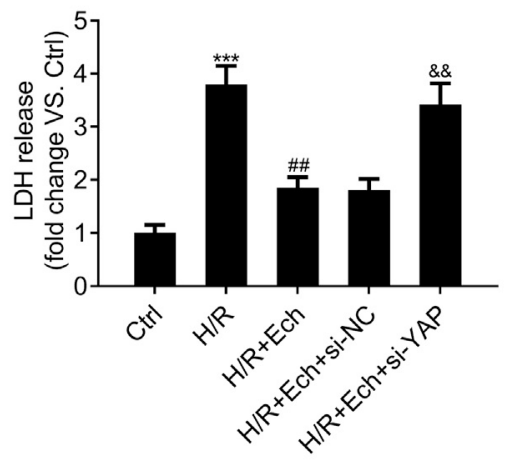

F

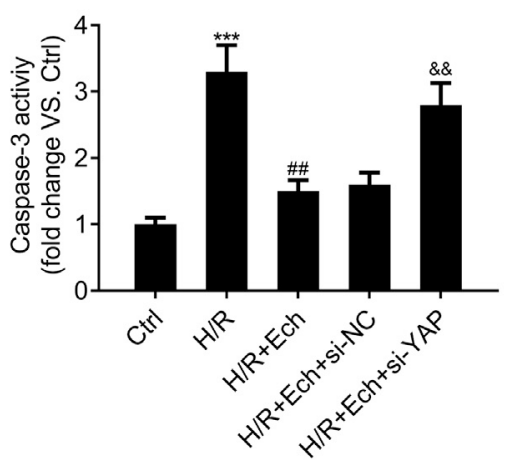

FIGURE 6 | Loss of YAP counteracts the protective effect of Ech on H/R-induced cell injury in vitro. (A, B) H9c2 cells were transfected with si-YAP and si-NC, and the silencing efficiency was determined by qRT-PCR and western blot analyses. After exposure to H/R, H9c2 cells were treated with Ech (10 $\mu \mathrm{m})$ for $24 \mathrm{~h}$ ( $n=3$ ). (C) Results of CCK-8 assay showed that silencing of YAP abrogated the protective effect of Ech on H/R-induced cell viability inhibition ( $n=3$ ). (D) The inhibitory effect of Ech on $\mathrm{H} / \mathrm{R}$-induced LDH release was blocked by YAP knockdown $(n=3)$. (E) Representative images and quantification of TUNEL staining showing that the protective effect of Ech on $\mathrm{H} / \mathrm{R}$-induced $\mathrm{H} 9 \mathrm{c} 2$ cell apoptosis was counteracted by si-YAP transfection $(n=3)$. (F) Analysis of caspase-3 activity showing the increased activity of caspase-3 in $\mathrm{H} 9 \mathrm{c} 2$ cells treated with si-YAP, $\mathrm{H} / \mathrm{R}$, and Ech relative to $\mathrm{H} 9 \mathrm{c} 2$ cells treated with $\mathrm{H} / \mathrm{R}$ and Ech $(n=3)$. ${ }^{\star \star \star} p<0.001 \mathrm{compared}$ with the si-NC and Ctrl groups; ${ }^{\# \#} p<0.01$ compared with the H/R group; ${ }^{\& \&} p<0.01$ compared with the $\mathrm{H} / \mathrm{R}+$ Ech + si-NC group.

2015). It is known that reduction of cardiomyocyte apoptosis is a crucial factor in improving myocyte contractile function.

Recently, there is emerging interest in the use of Ech because of its antioxidant and anti-inflammatory potential. The use of Ech as a natural antioxidant has been well established previously. Ech was shown to exert its antioxidant activity via an electron transfer and a proton transfer in aqueous solution and through the hydrogen atom transfer pathway in organic solution (Liang et al., 2018). Additionally, the anti-inflammatory effect of Ech has been also demonstrated. Ech treatment was shown to block lipopolysaccharide-induced elevation of prostaglandin E2 and interleukin-6 levels in RAW 264.7 macrophages (Fu et al., 2013).
Furthermore, Ech functioned as an activator of nuclear factor erythroid 2-related factor 2 to mitigate hepatoxicity and carbon tetrachloride-induced acute liver injury in mice, suggesting its antioxidant potential (Lin et al., 2017). Moreover, Ech reduced the levels of $\mathrm{LDH}$ and $\mathrm{CK}-\mathrm{MB}$, as well as the content of malondialdehyde, interleukin- 6 , and tumor necrosis factor- $\alpha$, and upregulated the activity of superoxide dismutase, thereby reducing myocardial infarct size and cardiomyocyte apoptosis (Tian et al., 2016). In the present study, our results showed that Ech administration mitigated MI/R-induced myocardial infarction and myocardial apoptosis in vivo and in vitro, thus confirming the cardioprotective effect of Ech. Our findings 
support the notion of Ech as a promising novel agent to remit MI/R injury.

$\mathrm{MI} / \mathrm{R}$ is a complicated biological process, which is mediated by several signaling mechanisms. To further characterize the protective effect of Ech on MI/R injury, we investigated the potential signaling mechanisms underlying the action of Ech on cardiomyocytes. Several lines of evidence suggest that Hippo/ YAP signaling plays a vital role in various biological processes, including cardiovascular development, cardiomyocyte regeneration, and autophagy (Plouffe et al., 2015). When the Hippo pathway is "switched on," MST1/2 is activated by adaptor protein salvador 1 -induced phosphorylation, which in turn phosphorylates and activates LATS1/2 (Mia and Singh, 2019). Activated LATS1/2 directly phosphorylate YAP, the core effector of Hippo signaling, followed by its cytoplasmic retention and then degradation via the proteosomal pathway (Kim and Jho, 2018). Conversely, non-phosphorylated YAP accumulates in the nucleus and functions as a transcriptional cofactor to participate in various cellular processes when Hippo signaling is "switched off" (Windmueller and Morrisey, 2015). Recently, dysregulation of Hippo/YAP signaling has been shown to be involved in the development of MI/R injury (Zhang and Del Re, 2017). For example, in a previous study, neurofibromin-2 activated by oxidative stress promoted cardiomyocyte apoptosis, induced the activation of MST1 and suppression of YAP, as well as protected against cardiomyocyte I/R injury, strongly suggesting a key role of Hippo signaling in $M I / R$ injury (Matsuda et al., 2016). Decreased expression of MST1, LATS1, and YAP/YAZ was also reported in AC16 cells subjected to H/R. Moreover, upregulation of YAP1 attenuated MI/R damage in AC16 human cardiomyocytes by co-activation of the Wnt/ $\beta$-catenin pathway (Khan et al., 2019). Additionally, blockage of the Hippo/YAP signaling pathway diminished the protective effect of melatonin on MI/R-induced cardiomyocyte death and mitochondrial damage, indicating the involvement of Hippo/YAP signaling in MI/R injury (Ma and Dong, 2019). Furthermore, activation of Hippo could antagonize YAPFoxO1 to restrain the expression of catalase and MnSOD, and then enhance oxidative stress-induced cell death during $M I / R$, suggesting that Hippo/YAP signaling mediates oxidative stressinduced MI/R damage (Shao et al., 2014). This finding highlights that targeting the Hippo/YAP pathway is regarded as a potential approach to reduce MI/R injury Mia et al. (2017). However, whether Hippo signaling mediates the protective effect of Ech on $\mathrm{MI} / \mathrm{R}$ injury remains poorly understood. In this study, our results

\section{REFERENCES}

Anderson, J. L., and Morrow, D. A. (2017). Acute myocardial infarction. N. Engl. J. Med. 376, 2053-2064. doi:10.1056/NEJMra1606915

Badalzadeh, R., Mokhtari, B., and Yavari, R. (2015). Contribution of apoptosis in myocardial reperfusion injury and loss of cardioprotection in diabetes mellitus. J. Physiol. Sci. 65, 201-215. doi:10.1007/s12576-0150365-8

Ferrari, R., Balla, C., Malagù, M., Guardigli, G., Morciano, G., Bertini, M., et al. (2017). Reperfusion damage-a story of success, failure, and hope. Circ. J. 81, 131-141. doi:10.1253/circj.CJ-16-1124 showed that Ech treatment inhibited the activation of Hippo/YAP signaling and induced the nuclear translocation of YAP, suggesting a strong correlation between Hippo/YAP signaling and MI/R injury. Moreover, silencing of YAP counteracted the protective effect of Ech on H/R-induced myocardial cell injury, thus providing evidence that Ech protects against MI/R damage at least partially by inducing Hippo/YAP pathway inactivation.

As a limitation of this study, YAP silencing was conducted only in in vitro experiments. We did not explore whether inhibition of Hippo/YAP signaling pathway can counteract the cardioprotective effect of Ech on $\mathrm{MI} / \mathrm{R}$ injury in vivo. In our future work, we will identify whether other mechanisms are also involved in the cardioprotective effect of Ech on MI/R injury.

\section{CONCLUSION}

Overall, our data revealed that Ech administration attenuated myocardial infarction and repressed cardiomyocyte apoptosis in in vivo and in vitro models of $\mathrm{MI} / \mathrm{R}$ injury. Subsequent mechanistic experiments demonstrated that Ech protected against $\mathrm{MI} / \mathrm{R}$ injury at least partially by suppressing the activation of Hippo/YAP signaling. Furthermore, we revealed the cardioprotective role and mechanism of action of Ech in MI/R injury, thus providing an experimental basis for its clinical use.

\section{DATA AVAILABILITY STATEMENT}

The raw data supporting the conclusions of this article will be made available by the authors, without undue reservation.

\section{ETHICS STATEMENT}

The animal study was reviewed and approved by the Animal Care and Use Committee of the Cangzhou Central Hospital.

\section{AUTHOR CONTRIBUTIONS}

JN, YnL, and XS performed the main experiments. YuL and YiL performed statistical analysis of all data. YaL contributed to conception, supervision, administration, and validation of this project. All authors reviewed the manuscript.

Fu, Y., Chen, J., Li, Y. J., Zheng, Y. F., and Li, P. (2013). Antioxidant and antiinflammatory activities of six flavonoids separated from licorice. Food Chem. 141, 1063-1071. doi:10.1016/j.foodchem.2013.03.089

Granger, D. N., and Kvietys, P. R. (2015). Reperfusion injury and reactive oxygen species: the evolution of a concept. Redox Biol. 6, 524-551. doi:10.1016/j.redox. 2015.08.020

Ji, S., Li, Z., Song, W., Wang, Y., Liang, W., Li, K., et al. (2016). Bioactive constituents of Glycyrrhiza uralensis (licorice): discovery of the effective components of a traditional herbal medicine. J. Nat. Prod. 79, 281-292. doi:10.1021/acs.jnatprod.5b00877

Khan, K., Makhoul, G., Yu, B., Schwertani, A., and Cecere, R. (2019). The cytoprotective impact of yes-associated protein 1 after ischemia-reperfusion 
injury in AC16 human cardiomyocytes. Exp. Biol. Med. 244, 802-812. doi:10. $1177 / 1535370219851243$

Kim, W., and Jho, E. H. (2018). The history and regulatory mechanism of the Hippo pathway. BMB Rep. 51, 106-118. doi:10.5483/bmbrep.2018.51.3.022

Kurian, G. A., Rajagopal, R., Vedantham, S., and Rajesh, M. (2016). The role of oxidative stress in myocardial ischemia and reperfusion injury and remodeling: revisited. Oxid. Med. Cell. Longev. 2016, 1-14. doi:10.1155/2016/1656450

Kwak, A. W., Choi, J. S., Lee, M. H., Oh, H. N., Cho, S. S., Yoon, G., et al. (2019). Retrochalcone echinatin triggers apoptosis of esophageal squamous cell carcinoma via ROS- and ER stress-mediated signaling pathways. Molecules 24 (22), 4055. doi:10.3390/molecules 24224055

Liang, M., Li, X., Ouyang, X., Xie, H., and Chen, D. (2018). Antioxidant mechanisms of echinatin and licochalcone A.. Molecules 24 (1), 3. doi:10. 3390/molecules 24010003

Lin, Y., Kuang, Y., Li, K., Wang, S., Ji, S., Chen, K., et al. (2017). Nrf2 activators from Glycyrrhiza inflata and their hepatoprotective activities against $\mathrm{CCl}_{4}$-induced liver injury in mice. Bioorg. Med. Chem. 25, 5522-5530. doi:10.1016/j.bmc. 2017.08.018

Ma, S., and Dong, Z. (2019). Melatonin attenuates cardiac reperfusion stress by improving OPA1-related mitochondrial fusion in a yap-hippo pathwaydependent manner. J. Cardiovasc. Pharmacol. 73, 27-39. doi:10.1097/fjc. 0000000000000626

Matsuda, T., Zhai, P., Sciarretta, S., Zhang, Y., Jeong, J. I., Ikeda, S., et al. (2016). NF2 activates Hippo signaling and promotes ischemia/reperfusion injury in the heart. Circ. Res. 119, 596-606. doi:10.1161/circresaha.116.308586

Mia, M. M., Chelakkot-Govindalayathil, L. A., and Singh, M. K. (2017). Targeting NF2-Hippo/Yap signaling pathway for cardioprotection after ischemia/ reperfusion injury. Ann. Transl. Med. 4, 545. doi:10.21037/atm.2016.11.85

Mia, M. M., and Singh, M. K. (2019). The Hippo signaling pathway in cardiac development and diseases. Front Cell. Dev. Biol. 7, 211. doi:10.3389/fcell.2019. 00211

Oh, H. N., Lee, M. H., Kim, E., Kwak, A. W., Seo, J. H., Yoon, G., et al. (2020). Dual inhibition of EGFR and MET by echinatin retards cell growth and induces apoptosis of lung cancer cells sensitive or resistant to gefitinib. Phytother. Res. 34, 388-400. doi:10.1002/ptr.6530

Ojha, S., Golechha, M., Kumari, S., Bhatia, J., and Arya, D. S. (2013). Glycyrrhiza glabra protects from myocardial ischemia-reperfusion injury by improving hemodynamic, biochemical, histopathological and ventricular function. Exp. Toxicol. Pathol. 65, 219-227. doi:10.1016/j.etp.2011.08.011

Plouffe, S. W., Hong, A. W., and Guan, K. L. (2015). Disease implications of the Hippo/YAP pathway. Trends Mol. Med. 21, 212-222. doi:10.1016/j.molmed. 2015.01.003

Reed, G. W., Rossi, J. E., and Cannon, C. P. (2017). Acute myocardial infarction. Lancet 389, 197-210. doi:10.1016/s0140-6736(16)30677-8
Shao, D., Zhai, P., Del Re, D. P., Sciarretta, S., Yabuta, N., Nojima, H., et al. (2014). A functional interaction between Hippo-YAP signalling and FoxO1 mediates the oxidative stress response. Nat. Commun. 5 (1), 3315. doi:10.1038/ ncomms 4315

Thygesen, K., Alpert, J. S., Jaffe, A. S., Chaitman, B. R., Bax, J. J., Morrow, D. A., et al. (2018). Fourth universal definition of myocardial infarction (2018). J. Am. Coll. Cardiol. 72, 2231-2264. doi:10.1016/j.jacc.2018.08.1038

Tian, X. H., Liu, C. L., Jiang, H. L., Zhang, Y., Han, J. C., Liu, J., et al. (2016). Cardioprotection provided by Echinatin against ischemia/reperfusion in isolated rat hearts. BMC Cardiovasc. Disord. 16, 119. doi:10.1186/s12872016-0294-3

Windmueller, R., and Morrisey, E. E. (2015). Hippo and cardiac hypertrophy: a complex interaction. Circ. Res. 117, 832-834. doi:10.1161/circresaha.115. 307546

Wu, H. J., Yang, J. Y., Jin, M., Wang, S. Q., Wu, D. L., Liu, Y. N., et al. (2015). Glycyrrhetinic Acid protects the heart from ischemia/reperfusion injury by attenuating the susceptibility and incidence of fatal ventricular arrhythmia during the reperfusion period in the rat hearts. Cell. Physiol. Biochem. 36, 741-752. doi:10.1159/000430134

Wu, M. Y., Yiang, G. T., Liao, W. T., Tsai, A. P., Cheng, Y. L., Cheng, P. W., et al. (2018). Current mechanistic concepts in ischemia and reperfusion injury. Cell. Physiol. Biochem. 46, 1650-1667. doi:10.1159/000489241

Yuan, X., Niu, H. T., Wang, P. L., Lu, J., Zhao, H., Liu, S. H., et al. (2015). Cardioprotective effect of licochalcone D against myocardial ischemia/ reperfusion injury in langendorff-perfused rat hearts. PLoS One 10, e0128375. doi:10.1371/journal.pone.0128375

Zhang, X., Zhu, P., Zhang, X., Ma, Y., Li, W., Chen, J. M., et al. (2013). Natural antioxidant-isoliquiritigenin ameliorates contractile dysfunction of hypoxic cardiomyocytes via AMPK signaling pathway. Mediat. Inflamm. 2013, 1-10. doi: $10.1155 / 2013 / 390890$

Zhang, Y., and Del Re, D. P. (2017). A growing role for the Hippo signaling pathway in the heart. J. Mol. Med. 95, 465-472. doi:10.1007/s00109-017-1525-5

Conflict of Interest: The authors declare that the research was conducted in the absence of any commercial or financial relationships that could be construed as a potential conflict of interest.

Copyright (c) $2021 \mathrm{Niu}, \mathrm{Li}$, Song, Liu, Li and Li. This is an open-access article distributed under the terms of the Creative Commons Attribution License (CC BY). The use, distribution or reproduction in other forums is permitted, provided the original author(s) and the copyright owner(s) are credited and that the original publication in this journal is cited, in accordance with accepted academic practice. No use, distribution or reproduction is permitted which does not comply with these terms. 